\title{
THREE NEW RENAL SIMULATORS FOR USE IN NUCLEAR MEDICINE
}

\author{
Marcos Dullius ${ }^{1,2 a}$, Mateus Fonseca ${ }^{2}$, Marcelo Botelho², Clêdison Cunha ${ }^{2}$, and Divanízia Souza ${ }^{2}$ \\ ${ }^{1}$ Universidade Federal da Fronteira Sul, Campus Cerro Largo-RS, Brazil, 97900-000 \\ ${ }^{2}$ Universidade Federal de Sergipe, São Cristóvão-SE, Brazil, 49100000
}

\begin{abstract}
Renal scintigraphy is useful to provide both functional and anatomic information of renal flow of cortical functions and evaluation of pathological collecting system. The objective of this study was develop and evaluate the performance of three renal phantoms: Two anthropomorphic static and another dynamic. The static images of the anthropomorphic phantoms were used for comparison with static renal scintigraphy with $99 \mathrm{mTc}-D M S A$ in different concentrations. These static phantoms were manufactured in two ways: one was made of acrylic using as mold a human kidney preserved in formaldehyde and the second was built with ABS (acrylonitrile butadiene styrene) in a 3D printer. The dynamic renal phantom was constructed of acrylic to simulate renal dynamics in scintigraphy with 99mTc-DTPA. These phantoms were scanned with static and dynamic protocols and compared with clinical data. Using these phantoms it is possible to acquire similar renal images as in the clinical scintigraphy. Therefore, these new renal phantoms can be very effective for use in the quality control of renal scintigraphy, and image processing systems.
\end{abstract}

\section{Introduction}

One way of ensuring quality control in nuclear medicine is through the use of phantoms during the tests required for practice of quality control. In accordance with the Brazilian standards, defined in CNEN-NN-3.05[1], and the documents of the IAEA (International Atomic Energy Agency) [2] it is recommended the use of phantoms suitable for realization of quality control of equipment and of image acquisition methods employed in nuclear medicine. Radioisotope renography is a form of kidney imaging involving radioisotopes. In nuclear medicine renal phantom are essentials to estimate the efficiency of systems in generating scintigraphic images to assess different clinical characteristics in renograms, as well as the ability of these systems to perform quantitative analyzes [3-5].

In view of the importance of quality control programs for image acquisition equipment, scintigraphic reconstruction programs used in nuclear medicine and the need of continuous training of nuclear medicine professionals, [6,7], the objective of this work was to develop two static renal phantoms and a dynamic renal phantom for use in nuclear medicine. These phantoms were scanned with static and dynamic protocols and compared with clinical data.

\footnotetext{
${ }^{\mathrm{a}}$ Corresponding author: divanizi@ufs.br
} 


\section{Methodology}

\subsection{Phantons for static renal scintigraphy}

For the development of a static renal anthropomorphic phantom using acrylonitrile butadiene styrene (ABS) it was used a chest tomographic imaging to obtaining the mold of the kidneys, as shown in Figure 1 (a). After create a 3D mesh via DICOM images generated from CT scans, the phantoms were making in a BFB 3D printer model TouchTM. It was used a free software for image reconstruction of the kidneys (Slicer, MeshLab, Netfabb Studio and BfB Axon). This technique allows the creation of anthropomorphic phantoms with external and internal structures, as shown in Figure 1 (b-c). This phantom is called here as ABS renal phantom.

Another static renal phantom was built using as mold a human kidney preserved in formaldehyde. The kidneys were modeled using alginate and autopolimerized acrylic, ensuring proper proportions and geometry of these organs. Figure 1 (d) shows the left and right static kidneys of the phantom.

In order to obtains the images, the phantoms were filled with $37 \mathrm{MBq}(1 \mathrm{mCi})$ of Tc-99m-DTPA diluted in water.

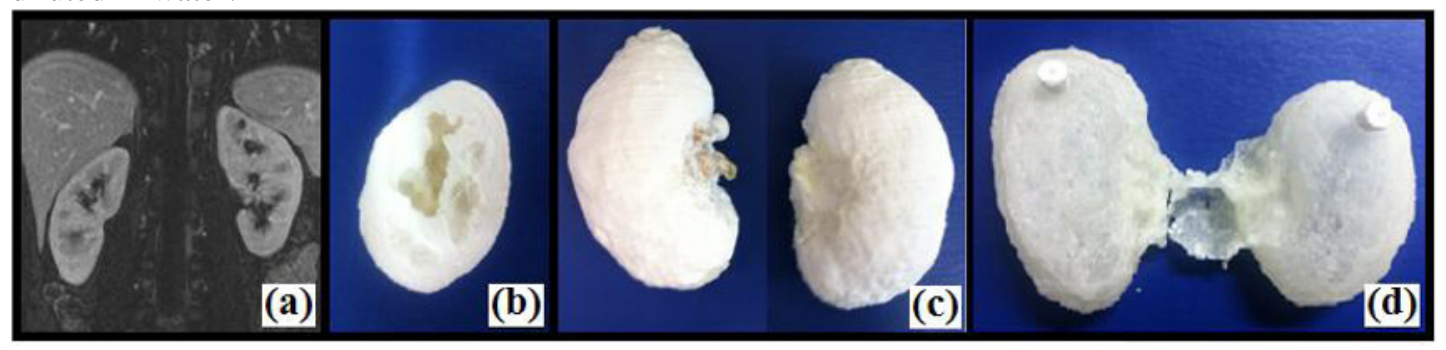

Figure 1. (a) TC used for 3D kidneys reconstruction. (b) Image showing the preservation of internal structures of the ABS renal phantom. (c) External view of the ABS renal phantom. (d) Static renal anthropomorphic phantom prepared with acrylic.

\subsection{Renal dynamic phantom}

The dynamic phantom for renal scintigraphy was made with acrylic and some other materials considering the average dimensions of the human renal system. Syringes on top of the kidneys enable the controlled injection of liquid. Hydraulic pumps assist in the controlled injection of liquid. A homogenizer completes this system in order to maintain the homogeneity of the mixture composed of water and radionuclide (tracer).

To enable the flow of the tracer the injection pumps are controlled by an electrical system, which in turn controls the time each pump is in operation. Changes in operating time of a pump imply in different rates for the radiopharmaceutical elimination from the phantom, allowing to obtaining different types of renogram. Images were acquired every 2 seconds for 1 minute (flow phase), followed by 15 -second images for 20 minutes (uptake phase). Figure 2 shows the dynamic renal phantom. 


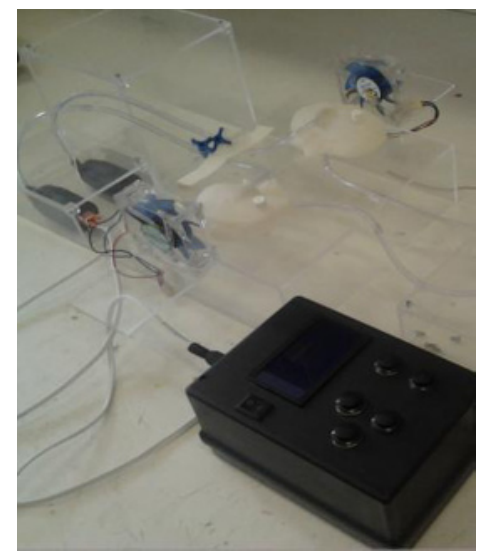

Figure 2. Phantom for dynamic renal scintigraphy.

\section{Results and Discussion}

\subsection{Static renal phantoms}

In Figures 3 (a) and (b) it can be observed images of the renal anthropomorphic static phantoms filled with $37 \mathrm{MBq}(1 \mathrm{mCi})$ of $99 \mathrm{mTc}-\mathrm{DMSA}$ dissolved in water.

In Figure 3 (a) images show a uniform homogeneous uptake in the cortical image performed with the acrilic phantom. Figure 3 (b) shows too images of homogeneous uptake, with small irregularities, resulting from the simulation of a preserved internal anatomy with ABS phantom. The zoom in these figures allow better observation of these details. In Figure 3 (c) we can see the image of a low renal uptake of the right kidney obtained using ABS phantom. A renal obstruction is characterized by a low renal uptake. In order to simulate the renal obstruction it was administered a low activity to the right kidney using $18.5 \mathrm{MBq}(500 \mu \mathrm{Ci})$ of $99 \mathrm{mTc}-\mathrm{DMSA}$.
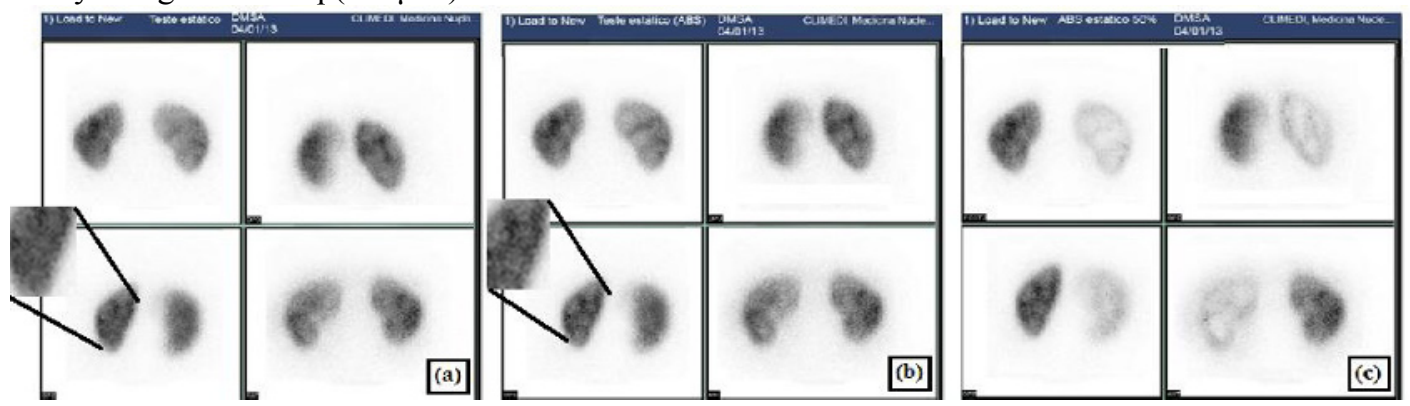

Figure 3. Images of scintigraphy obtained of static renal phantoms.

The static renal phantoms are able to simulate the renal function and generate images of static renal scintigraphy with $99 \mathrm{mTc}$-DMSA similar to those obtained from normal patients as those with renal obstruction. Therefore, static renal phantoms can help to instruct professionals in nuclear medicine.

\subsection{Dynamic renal phantom}

The dynamic phantom was calibrated to provide data on the elimination of the radioisotope by the kidneys according standards of normal renal scintigraphy. Figure 4 shows scintigraphic images of the phantom in a simulated dynamic renal scintigraphy. It can be observed a regular pattern of uptake and excretion. 
Scintigraphic images of the dynamic phantom presented normal default in the 99mTc-DTPA uptake phase. The renal cortex is homogeneous with similar appearance of health kidneys. The central image shows the bladder and kidneys with their selected areas to acquire the renograms. The uptake phase of the renograms is located at the bottom right in image. In the bottom left is shown the excretory phase.

The dynamic renal phantom showed, during the uptake phase (dynamic study), the renal cortex with homogeneous aspect, similar to that of health kidneys. Still, we can view a lower uptake in the left kidney, both in the uptake and excretory phase. This difference is due to the change in flow of tracer injection, since this injection is still done manually.

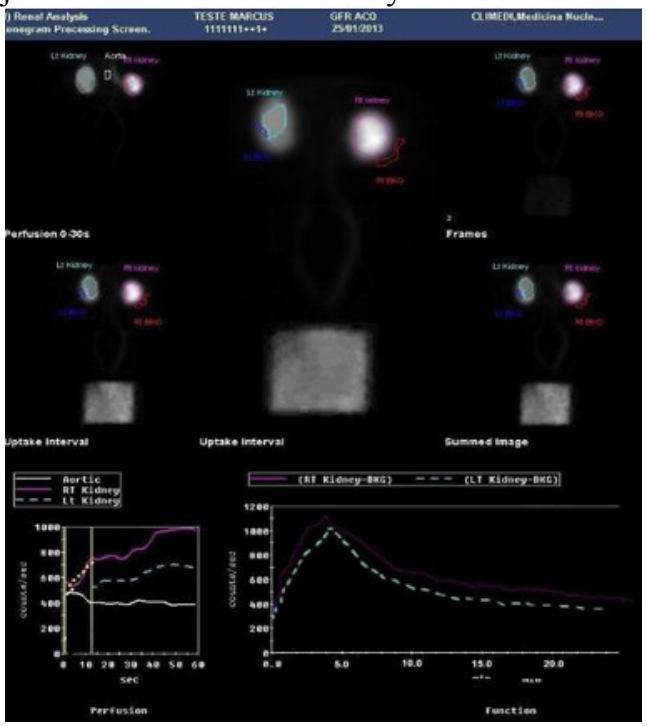

Figure 4. Image of dynamic renal scintigraphy (99mTc-DTPA) obtained using the dynamic renal phantom.

\section{Conclusions}

The evaluation of the phantoms in quality control processes of static scintigraphic images was made through the acquisition of static renal scintigraphy images with 99mTc-DMSA. The results of these tests were satisfactory, confirming that the use of these phantons can serve as a parameter for the quality control of equipment and scintigraphic imaging studies with the aim of clinical training in nuclear medicine for diagnosis of renal anomalies.

The use of dynamic renal phantom for quality control of gamma cameras by obtaining renograms can be done, because we observed that the patterns of scintigraphic image of the phantom showed a normal renal scintigraphy, representing kidneys with regular appearance.

\section{References}

1. CNEN-NE 3.05, Rio de Janeiro (1996).

2. IAEA - TECDOC-602, Vienna (1991).

3. J. O. Heikkinen, Phys. Med. Biol. 44 (1999).

4. J. O. Heikkinen, J. T. Kuikka, P. J. Rautio, J. Nucl. Med. 34 (2006).

5. A. S. M. Sabbir Ahmed, M. Demir, L. Kabasakal, I. Uslu. Med. Phys. 32 (2005).

6. M. A. Dullius, R. C. Fernandes, D. N. Souza, Waset, 80 (2011).

7. D. Debrun, F. The'rain, L. Nguyen, C. P. Le'ger, J. J. N. Visser, E. Busemann-sokole, iJCVI, 21, (2005). 